\title{
Effects of total replacement of dietary fish oil by vegetable oils on growth performance, nutritional quality and fatty acid profiles of rainbow trout (Oncorhynchus mykiss) at optimum and high temperature conditions
}

\author{
Yemdeki balık yağının bitkisel yağlarla tamamen değiştirilmesinin optimum \\ ve yüksek su sıcaklığında beslenen gökkuşağı alabalığının (Oncorhynchus \\ mykiss) büyüme performansı, besin kalitesi ve yağ asidi profili üzerine \\ etkileri
}

\author{
Seval Dernekbaşı ${ }^{\text {** }}$ • Ayşe Parlak Akyüz² • İsmihan Karayücel ${ }^{3}$ \\ 1 Sinop Üniversitesi, Su Ürünleri Fakültesi, Yetiştiricilik Anabilim Dalı, Akliman, Sinop \\ ${ }^{2}$ Kuzey Su Ürünleri San. ve Tic. Ltd. Şti. Bafra, Samsun \\ ${ }^{3}$ Sinop Üniversitesi, Su Ürünleri Fakültesi, Yetiştiricilik Anabilim Dalı, Akliman, Sinop \\ (D) https://orcid.org/0000-0001-5735-2486
(D) https://orcid.org/0000-0001-9099-4368
(D) https://orcid.org/0000-0003-2520-7545
}

\section{How to cite this paper:}

Dernekbaşı, S., Parlak Akyüz, A. \& Karayücel, I. (2021). Effects of total replacement of dietary fish oil by vegetable oils on growth performance, nutritional quality and fatty acid profiles of rainbow trout (Oncorhynchus mykiss) at optimum and high temperature conditions. Ege Journal of Fisheries and Aquatic Sciences, 38(2), 237-246. DOI: 10.12714/egejfas.38.2.14

\begin{abstract}
The present study investigated the effects of total replacement of dietary fish oil by different vegetable oils on growth performance, nutritional quality and fatty acid profiles of rainbow trout (Oncorhynchus mykiss) at optimum and high temperature conditions. Five iso-nitrogenous and iso-lipidic diets including $100 \%$ fish oil and vegetable oils were prepared for rainbow trout fingerlings with an average weight of $11.27 \pm 0.01 \mathrm{~g}$. After the fish were fed experimental diets for 9 weeks at the optimum temperature $\left(17.74 \pm 0.01^{\circ} \mathrm{C}\right)$, all groups were fed an FO diet containing only fish oil for 4-weeks at the upper optimum temperature conditions $\left(19.28 \pm 0.11^{\circ} \mathrm{C}\right)$. In both feeding trials, experimental fish were hand-fed ad libitium twice a day. Results showed that growth performance and feeding efficiency were significantly better $(p<0.05)$ in groups fed by VO-based diets compared to groups fed by FO based diet at optimum temperature. Survival was 100\% in CANO, SFO, CO PNO groups and $94.12 \pm 3.39 \%$ in FO (control) group at the end of the 9 weeks. Growth, feed consumption and survival of fish fed the upper-optimum temperature were significantly differed $(p<0.05)$. In particular, while the survival rate of the groups fed with vegetable oil-based diets at optimum temperature and then fed only fish oil remained $100 \%$, this rate decreased to $54.17 \pm 1.39 \%$ in the control group. Eicosapentaenoic acid (EPA, 20:5n-3), docosahexaenoic acid (DHA, 22:6n-3) and n-3 PUFA of fish fed by VO based diets were low. When all groups were fed only FO diet for 4 weeks at upper-optimum temperatures, EPA, DHA and n-3 PUFA ratios increased. In addition, after the upper-optimum temperature trial, increases in the nutritional quality indices of fish meat were also detected.

Keywords: Salmonids, feed formulation, growth metrics, compensatory baiting

Öz: Bu çalışmada, balık yağının farklı bitkisel yağlarla toplam ikamesinin, optimum ve yüksek sıcakıık koşullarında gökkuşağı alabalı̆ııın (Oncorhynchus mykiss) büyüme performansı, beslenme kalitesi ve yağ asidi profilleri üzerindeki etkileri araştırımıştır. Ortalama $11,27 \pm 0,01 \mathrm{~g}$ ağırlıktaki gökkusağı alabalığı yavruları için \%100 balık yağı ve bitkisel yağlardan oluşan bes izo-nitrojenli ve izo-lipidik diyet hazırlanmıștır. Balıklar optimum sıcaklıkta $\left(17,74 \pm 0,01^{\circ} \mathrm{C}\right) 9$ hafta deneysel diyetlerle beslendikten sonra, tüm gruplara optimum üstü sıcaklık koşullarında $\left(19,28 \pm 0,11^{\circ} \mathrm{C}\right) 4$ hafta boyunca sadece balık yağı içeren bir FO (kontrol) diyeti verilmiştir. Her iki besleme denemesinde de deneysel balıklar günde iki kez elle doyuncaya kadar beslenmiştir. Sonuçlar, optimum sıcaklıkta FO bazlı diyetle beslenen gruplara kıyasla, VO bazlı diyetlerle beslenen gruplarda büyüme performansı ve yemleme veriminin önemli ölçüde daha iyi $(p<0,05)$ olduğunu göstermisțir. Yassama oranı, 9 hafta sonunda CANO, SFO, CO PNO gruplarında $\% 100$ ve FO (kontrol) grubunda $\% 94,12 \pm 3,39$ olarak tespit edilmiştir. Optimum sıcaklıkta beslenen balıkların büyümesi, yem tüketimi ve hayatta kalması önemli ölçüde farklllık göstermişstir ( $p<0,05)$. Özellikle bitkisel yağ bazlı diyetlerle optimum sıcaklıkta beslenen ve daha sonra sadece balık yağı ile beslenen grupların hayatta kalma oranı \% 100 kalıken, kontrol grubunda bu oran $\% 54,17 \pm 1,39$ 'a gerilemiştir. VO bazlı diyetlerle beslenen balıkların eikosapentaenoik asit (EPA, 20:5n-3), dokosaheksaenoik asit (DHA, 22:6n-3) ve n-3 PUFA düš̈ük olduğu tespit edilmiștir. Tüm gruplara optimum sıcaklıklarda 4 hafta boyunca sadece FO diyeti verildiğinde EPA, DHA ve n-3 PUFA oranları artmışıı. Ayrıca optimum üst sıcaklık denemesinin ardından balık etinin besin kalitesi endekslerinde de artışlar tespit edilmiştir.

Anahtar kelimeler: Alabalıklar, yem formülasyonu, büyüme ölçümleri, telafi yemlemesi
\end{abstract}

\section{INTRODUCTION}

In the fish feed industry, fish oil costs increased due to the decline in fish stocks and rising demand (Izquierdo et al., 2005; Şahan et al., 2017), and this increase in fish oil prices was reflected in feed costs. In fish feeds, it is very important to prepare a balanced ration to meet the needs of fish. Balanced feed rations play an important role in implementing most of the physiological functions for fish. Creating an economical and balanced ration for feed, finding alternative fish oil sources that can meet the need for the similar fish fatty acids without causing metabolic deterioration and health impairment will reduce the cost of the market sector and utility the economy (Sahan et al., 2016). 
Due to the fact that the production of vegetable oils is easy and inexpensive, it can be used as an alternative to fish oil in different proportions and it is generally accepted by the researchers that there is no negative effect on growth of fish (Torstensen et al., 2000; Caballero et al., 2002; Yildiz et al., 2015; Yıldiz et al., 2018). These rates can be up to $100 \%$ in freshwater fish while it was determined as $60 \%$ in marine fish (Izquierdo et al., 2005). Most vegetable oils are rich in unsaturated $18 \mathrm{C}$ fatty acids such as oleic (18:1n-9), linoleic $(18: 2 n-6)$ and alpha-linolenic acids (a-18:3n-3) but are poor sources for $n-3$ HUFAs, so the flesh of farmed fish given feeds containing high concentrations of vegetable oils may contain a limited amount of n-3 HUFAs (Sonu, 2018). This dilemma has led to the development of using only a fish oil diet to restore n-3 PUFA levels in fish after a growth period on vegetable oil (VO) based diet. Feeding fish only fish oil after grow-out period based on vegetable oil diet is one strategy to restore eicosapentaenoic acid (EPA) and docosahexaenoic acid (DHA) levels (Codabaccus et al., 2013).

Rainbow trout is defined as a species of cold water fish, and especially in trout farming, an increase in water temperature can lead to a decrease in feed intake, growth rate and survival rate and an increase in disease incidence. In recent years, researchers have accelerated the studies on the use of vegetable oils showing antioxidant features as an alternative antioxidant in order to prevent diseases, increase growth performance and strengthen the immune system. Antioxidation is important for nutrition because of reducing physiological stress in organs and cells (Bayaz, 2014). Studies showed that these substances have quite beneficial effects and their main advantage is that they are of natural origin and do not pose any threat to human, fish health or the environment. (Gabor et al., 2012).

For this reason, the current study, after feeding rainbow trout with vegetable oil-based and fish-oil diets at optimum temperature, in all groups, fed by diets containing only fish oil at upper-optimum temperature for a certain period of time, in particular, it was tried to determine how it would affect survival and growth. In addition, it is aimed to regain the nutritional quality indexes that decreased as a result of feeding with $n-3$ PUFA and vegetable oils in a certain period after the end of the growth period by using fish oil diets.

\section{MATERIAL AND METHODS}

\section{Experimental diets and feeding}

Five experimental diet groups were formulated to be isonitrogenous (48\%) and iso-lipidic (17\%), containing different lipid sources (Table 1).

Fish oil and feed ingredients were obtained from a local fish feed manufacturer (Sibal A.Ş. Black Sea Feed, Sinop, Turkey). Canola and peanut oils were purchased from commercial companies (Tokul Tarım A.Ş., İzmir/Turkey and Başpınar Toprak Mah. ve Nak. Ltd. Şti., Osmaniye, Turkey, respectively). Sunflower and corn oil were purchased from a supermarket (ŞOK Marketler T.A.Ş., Sinop, Turkey). Diets were prepared by a laboratory pelleting machine after 35- $40 \mathrm{~g}$ of distilled water was added into $1000 \mathrm{~g}$ mixture of ingredients and the pellets were dried at $40^{\circ} \mathrm{C}$ for $12 \mathrm{~h}$. Diets were crushed to the approximate size of $1-2 \mathrm{~mm}$ and kept at $-20^{\circ} \mathrm{C}$.

Table 1. Ingredients ( $\mathrm{g} / \mathrm{kg}$ ) and proximate composition (\%) of the experimental diets containing the different vegetable oils and fish oil

\begin{tabular}{|c|c|c|c|c|c|}
\hline & & Exp & rimenta & al diets & \\
\hline & $\begin{array}{c}\text { FO } \\
\text { (Control) }\end{array}$ & CANO & co & SFO & PNO \\
\hline Ingredients ( $\left.\mathrm{g} \mathrm{kg}^{-1}\right)$ & & & & & \\
\hline Fish meal & 350 & 350 & 350 & 350 & 350 \\
\hline Extracted soybean meal & 250 & 250 & 250 & 250 & 250 \\
\hline Wheat flour & 130 & 130 & 130 & 130 & 130 \\
\hline Corn protein & 146 & 146 & 146 & 146 & 146 \\
\hline Fish oil & 120 & 0 & 0 & 0 & 0 \\
\hline Canola oil & 0 & 120 & 0 & 0 & 0 \\
\hline Corn oil & 0 & 0 & 120 & 0 & 0 \\
\hline Sunflower oil & 0 & 0 & 0 & 120 & 0 \\
\hline Peanut oil & 0 & 0 & 0 & 0 & 120 \\
\hline Vitamin premix( $\left(^{*}\right)$ & 2 & 2 & 2 & 2 & 2 \\
\hline Mineral premix(*) & 2 & 2 & 2 & 2 & 2 \\
\hline Proximate Compositio & & & & & \\
\hline Moisture & 4.08 & 4.34 & 3.69 & 3.63 & 4.81 \\
\hline Protein & 47.28 & 47.74 & 47.69 & 47.63 & 47.24 \\
\hline Lipid & 17.71 & 17.23 & 17.69 & 17.59 & 17.29 \\
\hline Ash & 9.74 & 9.55 & 9.22 & 9.39 & 9.28 \\
\hline $\mathrm{NFE}+\mathrm{Crude}$ fiber & 21.19 & 21.14 & 21.71 & 21.79 & 21.38 \\
\hline Gross energy $\left(\mathrm{kJg}^{-1}\right)$ & 21.75 & 21.76 & 21.95 & 21.82 & 21.58 \\
\hline
\end{tabular}

"Vitamin-mineral premix (mg/kg premix): vitamin A, 210000 IU; Vitamin $\mathrm{D}_{3}, 35000 \mathrm{IU}$; vitamin E, 7000 $\mathrm{mg}$; vitamin $\mathrm{K}_{3}, 322 \mathrm{mg}$; vitamin $\mathrm{B}_{1}, 588 \mathrm{mg}$; vitamin $\mathrm{B}_{2}, 252 \mathrm{mg}$; vitamin $\mathrm{B}_{6}, 294 \mathrm{mg}$; vitamin $\mathrm{B}_{12}, 826$ mcg; niacin, $1400 \mathrm{mg}$; biotin, $7583 \mathrm{mcg} ; 182 \mathrm{mg}$ folic acid, pantothenic acid, $1722 \mathrm{mg}$; inositol, 17220 $\mathrm{mg}$; vitamin C, $933.31 \mathrm{mg}$; Ca, $1414 \mathrm{mg}$. NFE+Crude fiber=100-(\%protein+\%lipid+\%ash+\%moisture)

The experiment was conducted in triplicates in randomly assigned tanks. During 9 weeks of optimum temperature and 4 weeks of the upper-optimum temperature, fish in all groups were hand fed twice a day (at 09:00 am and 15:00 pm) to apparent satiety under a natural light regime.

\section{Fish facilities and sampling}

Rainbow trout fingerlings were obtained from a commercial trout farm (Kuzey Su Ürünleri Inc., Samsun, Turkey) and acclimated in the Research and Application Center of Sinop University, Fisheries Faculty (Sinop, Turkey) for one week before the start of the experiment. Fish were stocked in centrally drained three 4000 I rectangle fiberglass tanks in a flow-through water system in an indoor facility during acclimatization. After acclimatization, fish (mean weight of $11.27 \pm 0.01 \mathrm{~g}$ ) were fasted for a day, weighted and 

optimum -and high temperature conditions

randomly distributed among fifteen fiberglass circular tanks (approximately, the water volume of $300-\mathrm{L} ; 60 \mathrm{~cm}$ in high; 80 $\mathrm{cm}$ in diameter) at a density of 17 fish per tank. Water inflow was adjusted to $4 \mathrm{l} / \mathrm{min}$ and supplemental aeration was provided via airstone diffusers. The fish were individually weighted at the beginning and end of the experiment with a $0.01 \mathrm{~g}$ sensitive electronic balance. The water quality parameters were monitored on a weekly basis and the following parameters were recorded. In this study was measured as average temperature of $17.74 \pm 0.01^{\circ} \mathrm{C}$, dissolved oxygen of $6.06 \pm 0.04 \mathrm{mg}^{-1}$ and $\mathrm{pH} 8.21$. At the beginning of the experiment, twenty fish were sacrificed, weighed and fish fillets were homogenized for analysis. At the end of the optimum temperature trial and the upper-optimum temperature trial, five fish from each tank were sampled and fish fillets were homogenized. Homogenized fish fillet samples were stored at $-80^{\circ} \mathrm{C}$ until used to proximate and fatty acid analysis.

\section{Upper-optimum temperature}

Fish used in the optimum temperature experiment were used in the experiment. The fish remaining after the optimum temperature trial were weighed. A new trial was created to be fed only the control diet for 4 weeks at the upper-optimum temperature. After this point, all fish were fed by a control diet (Table 1), which had the same composition as the FO diet used in the optimum temperature trial. In the upper-optimum temperature trial, the average water temperature was $19.28 \pm 0.11^{\circ} \mathrm{C}$ during 4 weeks. At the end of the trial, fish were individually weighed and five fish from each tank were randomly selected, euthanized by excess anesthetic (clove oil) and stored at $-80^{\circ} \mathrm{C}$ for future analysis.

\section{Proximate and fatty acid analysis}

The analyses of proximate composition on feed and fillet were performed according to the standard methods of $A O A C$ (1995): dry matter after drying in an oven at $105^{\circ} \mathrm{C}$ until constant weight, crude protein ( $\mathrm{Nx6.25)}$ by Kjeldhal method after acid digestion, ash content by incineration in a muffle furnace at $550^{\circ} \mathrm{C}$ for $12 \mathrm{~h}$, crude lipid after extraction with petroleum ether by the Soxhlet method.

Total lipid was determined by modified Bligh and Dyer Method (Hanson and Olley, 1963). $0.25 \mathrm{~g}$ of extracted oil from fish fillets and diets was dissolved by adding $4 \mathrm{ml}$ of heptane and $0.4 \mathrm{ml}$ of $2 \mathrm{~N} \mathrm{KOH}$ was added. This mixture was stirred in vortex for 2 minutes, then centrifuged at $5000 \mathrm{rpm}$ for 5 minutes. After centrifugation, $1.5-2 \mathrm{ml}$ of the heptane phase was collected and transferred to glass tubes for GC/MS analysis. The injection of samples into the device was performed with the autosampler Al 1310. Samples were analyzed by Thermo Scientific ISQ LT model GC/MS gas chromatography by spectrometer. For this analysis, with $0.25 \mu \mathrm{m}$ film thickness was used a Trace Gold TG-WaxMS capillary column (Thermo Scientific code: 26088-1540) in $0.25 \mu \mathrm{m}$ inner diameter and $60 \mu \mathrm{m}$ length. The injection block temperature was adjusted to $240^{\circ} \mathrm{C}$ and the column temperature program to be increased from $100^{\circ} \mathrm{C}$ to $240^{\circ} \mathrm{C}$. Helium gas (1 $\mathrm{ml} / \mathrm{min}$ ) was used as a carrier gas and 1:20 split ratio was applied. The MS unit (ISQ LT) was used in electron ionization mode. Fatty acids are defined by comparing the standard FAME mixture of 37 components [Chem-Lab Fame mix (37C) standard solution; Art. Nr. CL40. 13093; Lot Nr. 221.561.102.100] with respect to their arrival time.

\section{The nutritional quality indexes of the fillet lipids}

In the study, the nutritional quality indexes of fillet lipids were calculated according to the formulas below (Dagtekin et al., 2017; Yu et al., 2018; Kocatepe et al., 2019).

Index of Atherogenicity $(\mathrm{Al})=\left[\mathrm{C} 12: 0+\left(4^{*} \mathrm{C} 14: 0\right)+\right.$ C16:0] / ( (LMUFA + n-6 PUFA + n-3 PUFA)

Index of Thrombogenicity $(\mathrm{TI})=(\mathrm{C} 14: 0+\mathrm{C} 16: 0+\mathrm{C} 18: 0) /$ $\left(0.5^{\star}\right.$ EMUFA + 0.5*n-6 PUFA + $3^{*} n-3$ PUFA $)+(n-3$ PUFA $/ n-6$ PUFA)

Flesh Lipid Quality Index (FLQ) $=(C 20: 5$ n3 + C22:6 n3 / Etotal FA)*100

Hypocholesterolemic / hypercholesterolemic $(\mathrm{h} / \mathrm{H})=$ $(C 18: 1 n-9+C 18: 2 n-6+C 20: 4 n-6+C 18: 3 n-3+C 20: 5 n-3+$ C22:5n-3 + C22:6n-3) / (C14:0 + C16:0)

\section{Statistical analysis}

Anderson-Darling and Levene's tests were used for homogeneity of variances and equality of variance of groups, respectively. The effects of dietary oils on muscle fatty acid compositions and the significance of differences in growth between control and treated groups were analyzed using oneway analysis of variance (ANOVA), followed by Tukey's method for multiple comparisons. Arcsine square root transformations of percentage data were conducted for homogeneity of variances before statistical analysis. Differences were considered significant when $p<0.05$. Analyses were performed using Minitab 17 software for Windows.

\section{RESULTS}

\section{Fatty acid composition of the diets}

Fatty acid (FA) composition of fillets reflected the fatty acid composition of diets, although the differences in fatty acid composition of fish in the groups was less than the differences in fatty acid composition among their respective diets. The fatty acid composition of the experimental diets was shown in Table 2. 
Table 2. Fatty acid composition of the experimental diets (total fatty acids \%)

\begin{tabular}{|c|c|c|c|c|c|}
\hline & \multicolumn{5}{|c|}{ Experimental Diets } \\
\hline & FO (Control) & CANO & SFO & CO & PNO \\
\hline C14:0 & $5.67 \pm 0.04^{\mathrm{a}}$ & $1.28 \pm 0.00^{d}$ & $1.50 \pm 0.02^{\mathrm{a}}$ & $1.30 \pm 0.02^{\mathrm{d}}$ & $1.42 \pm 0.00^{\circ}$ \\
\hline C15:0 & $1.09 \pm 0.04^{\mathrm{a}}$ & $0.17 \pm 0.00^{b}$ & $0.21 \pm 0.00^{b}$ & $0.19 \pm 0.00^{b}$ & $0.19 \pm 0.00^{b}$ \\
\hline C16:0 & $13.51 \pm 0.14^{b}$ & $12.58 \pm 0.16^{c}$ & $12.32 \pm 0.13^{c}$ & $10.87 \pm 0.18^{d}$ & $14.96 \pm 0.11^{\mathrm{a}}$ \\
\hline C17:0 & $1.35 \pm 0.08^{a}$ & $0.25 \pm 0.05^{b}$ & $0.23 \pm 0.00^{b}$ & $0.23 \pm 0.02^{b}$ & $0.23 \pm 0.00^{b}$ \\
\hline C18:0 & $6.27 \pm 0.02^{c}$ & $7.29 \pm 0.07^{b}$ & $8.35 \pm 0.09 \mathrm{a}$ & $4.65 \pm 0.01^{d}$ & $6.36 \pm 0.06^{c}$ \\
\hline C20:0 & $0.37 \pm 0.08^{a}$ & $0.05 \pm 0.00^{\mathrm{b}}$ & $0.05 \pm 0.00^{b}$ & $0.13 \pm 0.01^{b}$ & $0.05 \pm 0.00^{b}$ \\
\hline C22:0 & $1.54 \pm 0.08^{c}$ & $4.86 \pm 0.00^{a}$ & $1.96 \pm 0.01^{b}$ & $1.05 \pm 0.01^{d}$ & $0.62 \pm 0.01 \mathrm{e}$ \\
\hline C24:0 & $0.49 \pm 0.02^{\mathrm{a}}$ & $0.05 \pm 0.00^{b}$ & $0.04 \pm 0.00^{b}$ & $0.05 \pm 0.01^{b}$ & $0.05 \pm 0.00^{b}$ \\
\hline$\Sigma$ SFA & $30.27 \pm 010^{\mathrm{a}}$ & $26.52 \pm 0.04^{b}$ & $24.65 \pm 0.01^{c}$ & $18.45 \pm 0.19 \mathrm{e}$ & $23.87 \pm 0.05^{\mathrm{d}}$ \\
\hline C14:1 & $0.38 \pm 0.02^{\mathrm{a}}$ & $0.05 \pm 0.00^{b}$ & $0.06 \pm 0.00^{b}$ & $0.06 \pm 0.01^{b}$ & $0.06 \pm 0.00^{b}$ \\
\hline C15:1 & $0.19 \pm 0.01^{a}$ & $0.03 \pm 0.00^{b}$ & $0.03 \pm 0.00^{b}$ & $0.03 \pm 0.00^{\mathrm{b}}$ & $0.03 \pm 0.00^{b}$ \\
\hline C16:1 & $6.72 \pm 0.04^{a}$ & $1.86 \pm 0.04^{c}$ & $1.86 \pm 0.04^{c}$ & $1.74 \pm 0.02^{\mathrm{d}}$ & $1.96 \pm 0.03^{b}$ \\
\hline C17:1 & $0.58 \pm 0.04^{a}$ & $0.16 \pm 0.01^{b}$ & $0.14 \pm 0.00^{b}$ & $0.16 \pm 0.0^{b}$ & $0.15 \pm 0.00^{b}$ \\
\hline C18:1 n-9c & $11.81 \pm 0.53^{d}$ & $32.92 \pm 0.30^{a}$ & $22.13 \pm 0.02^{c}$ & $31.84 \pm 0.56^{b}$ & $21.50 \pm 0.14 c$ \\
\hline C18:1 n-9t & $3.33 \pm 0.11^{b}$ & $2.06 \pm 0.55^{c}$ & $1.49 \pm 0.05^{\mathrm{cd}}$ & $4.32 \pm 0.11^{\mathrm{a}}$ & $1.37 \pm 0.01^{\mathrm{d}}$ \\
\hline C20:1 & $3.05 \pm 0.06^{b}$ & $3.09 \pm 0.00^{b}$ & $1.73 \pm 0.04^{d}$ & $3.40 \pm 0.04^{a}$ & $1.93 \pm 0.01^{c}$ \\
\hline C20:1 n-9 & $2.90 \pm 0.06^{a}$ & $1.65 \pm 0.03^{c}$ & $1.90 \pm 0.03^{b}$ & $1.61 \pm 0.01^{c}$ & $1.86 \pm 0.01^{b}$ \\
\hline C24:1 & $1.23 \pm 0.05^{\mathrm{a}}$ & $0.35 \pm 0.00^{c}$ & $0.39 \pm 0.00^{c}$ & $0.64 \pm 0.03^{b}$ & $0.39 \pm 0.00 c$ \\
\hline EMUFA & $30.25 \pm 0.36^{c}$ & $42.15 \pm 0.34^{b}$ & $29.72 \pm 0.09 c$ & $43.79 \pm 0.72^{\mathrm{a}}$ & $29.23 \pm 0.16^{c}$ \\
\hline C18:2n-6c & $11.26 \pm 0.30^{c}$ & $20.92 \pm 0.32^{b}$ & $37.88 \pm 0.19 a$ & $22.08 \pm 1.10^{b}$ & $37.55 \pm 0.12^{\mathrm{a}}$ \\
\hline C18:2 n-6t & $0.51 \pm 0.02^{\mathrm{a}}$ & $0.08 \pm 0.01^{b}$ & $0.06 \pm 0.00^{b}$ & $0.08 \pm 0.01^{b}$ & $0.06 \pm 0.00^{b}$ \\
\hline C18:3 n-3 & $1.62 \pm 0.08^{b}$ & $3.20 \pm 0.05^{a}$ & $1.09 \pm 0.01^{d}$ & $1.66 \pm 0.02^{b}$ & $1.46 \pm 0.02^{c}$ \\
\hline C18:3 n-6 & $4.15 \pm 0.02^{c}$ & $3.58 \pm 0.01^{\mathrm{d}}$ & $3.27 \pm 0.03^{\mathrm{e}}$ & $10.74 \pm 0.21^{a}$ & $4.45 \pm 0.02^{\mathrm{b}}$ \\
\hline $\mathrm{C} 20: 2$ & $0.65 \pm 0.04^{a}$ & $0.14 \pm 0.01^{c}$ & $0.13 \pm 0.01^{c}$ & $0.22 \pm 0.00^{b}$ & $0.16 \pm 0.01^{c}$ \\
\hline C20:3 n-6 & $0.44 \pm 0.02$ & nd & nd & nd & nd \\
\hline C20:4 n-6 & $1.54 \pm 0.08^{a}$ & $0.22 \pm 0.01^{b}$ & $0.22 \pm 0.00^{b}$ & $0.18 \pm 0.00^{b}$ & $0.22 \pm 0.00^{b}$ \\
\hline C20:5n-3 & $8.01 \pm 0.19 \mathrm{a}$ & $1.49 \pm 0.03^{b}$ & $1.28 \pm 0.03^{b c}$ & $1.19 \pm 0.01^{c}$ & $1.31 \pm 0.01^{b c}$ \\
\hline C22:2 & $0.18 \pm 0.01^{a}$ & $0.15 \pm 0.00^{b}$ & $0.13 \pm 0.00^{c}$ & $0.09 \pm 0.01 \mathrm{e}$ & $0.10 \pm 0.00^{d}$ \\
\hline C22:6 n-3 & $10.83 \pm 0.13^{a}$ & $1.37 \pm 0.01^{b}$ & $1.38 \pm 0.02^{b}$ & $1.28 \pm 0.01^{b}$ & $1.41 \pm 0.02^{b}$ \\
\hline EPUFA & $39.16 \pm 0.25 c$ & $31.11 \pm 0.30 \mathrm{e}$ & $45.42 \pm 0.09 \mathrm{~b}$ & $37.49 \pm 0.87^{d}$ & $46.71 \pm 0.12^{\mathrm{a}}$ \\
\hline$\Sigma n-3$ PUFA & $20.45 \pm 0.23^{a}$ & $6.05 \pm 0.02^{b}$ & $3.74 \pm 0.06^{d}$ & $4.12 \pm 0.02^{c}$ & $4.18 \pm 0.00 \mathrm{c}$ \\
\hline 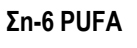 & $17.88 \pm 0.43^{d}$ & $24.79 \pm 0.30 c$ & $41.43 \pm 0.16^{a}$ & $33.07 \pm 0.89 \mathrm{~b}$ & $42.28 \pm 0.11^{a}$ \\
\hline$n-3 / n-6$ & $1.14 \pm 0.04^{a}$ & $0.24 \pm 0.00^{b}$ & $0.09 \pm 0.00 \mathrm{c}$ & $0.12 \pm 0.00 c$ & $0.10 \pm 0.00 c$ \\
\hline EPA/DHA & $0.74 \pm 0.01^{b}$ & $1.09 \pm 001^{a}$ & $0.93 \pm 0.00 \mathrm{a}$ & $0.93 \pm 0.00^{a}$ & $0.93 \pm 0.01^{a}$ \\
\hline
\end{tabular}

nd: not detected, FO: Fish oil, CANO: Canola oil, CO: Corn oil, SFO: Sunflower oil, PNO: Peanut oil. SFA: Saturated fatty acids, MUFA: Monounsaturated fatty acids, PUFA: Polyunsaturated fatty acids, DHA: Docosahexaenoic acid, EPA: Eicosapentaenoic acid, Values are means of three determinations. 


\section{Growth performance, feeding efficiency and survival}

In the optimum temperature, significant differences were detected in both the growth performance and feeding efficiencies between FO and VO based diet trials (Table 3). The weight of all fish increased by approximately 30 to 43 fold and specific growth rate (SGR) varied between 2.27 and $2.69 \%$ day $^{-1}$ while the weight gains (WG) were between 30.73 and $42.38 \mathrm{~g}$. Feed conversion ratios (FCR), hepatosomatic index (HSI) were not significantly different among the groups and ranged from 1.29-1.61 and 1.39$2.08 \%$, respectively. The survival was high among the groups fed by VO based diets (100\%), but it was $94.12 \pm 3.39 \%$ in the groups fed by $\mathrm{FO}$ based diet. No significant differences were detected among the trial groups in terms of vicerosomatic index (VSI) values $(p>0.05)$.
In the upper-optimum temperature, significant differences in the survival, growth performance, FCR and other body parameters were detected among all fish after fed by FO (control) diet for four weeks (Table 3; $p>0.05$ ). At the end of the trial, fish in FO group had significant lower final body weight (FBW), weight gain (WG), FCR and SGR (43.40 g, $1.73 \mathrm{~g}, 2.05$ and 0.14 , respectively) compared to CANO (55.39 g, $4.52 \mathrm{~g}$ and 0.30), SFO (74.30 g, $23.43 \mathrm{~g}$ and 1.33), CO (67.12 g, $16.58 \mathrm{~g}$ and 1.01) and PNO groups (77.76 g, $27.09 \mathrm{~g}$ and 1.52). The lowest HSI value was detected in the SFO group, similarly among in CANO, CO and PNO groups, while it was $1.45 \%$ in FO group. The VSI value was significantly higher in SFO and CO groups than the FO, CANO and PNO groups ( $p>0.05)$. The survival rate was significantly lower in the FO group, while it was $100 \%$ in the other groups $(p>0.05)$.

Table 3. Growth performance, feed efficiency and other body parameters of rainbow trout fingerlings fed optimum and upper-optimum temperature

\begin{tabular}{|c|c|c|c|c|c|c|c|c|c|c|}
\hline & \multicolumn{5}{|c|}{ Optimum Temperature $\left(17^{\circ} \mathrm{C}\right)$} & \multicolumn{5}{|c|}{ Upper-Optimum Temperature $\left(19^{\circ} \mathrm{C}\right)$} \\
\hline & FO & CANO & SFO & CO & PNO & FO & CANO & SFO & $\mathrm{CO}$ & PNO \\
\hline IBW (g) & $11.26 \pm 0.01$ & $11.27 \pm 0.02$ & $11.26 \pm 0.01$ & $11.27 \pm 0.05$ & $11.29 \pm 0.04$ & $41.67 \pm 0.80$ & $50.86 \pm 4.54$ & $50.87 \pm 2.42$ & $50.54 \pm 0.46$ & $50.67 \pm 1.01$ \\
\hline FBW (g) & $42.17 \pm 0.59^{a}$ & $52.70 \pm 6.68^{b}$ & $51.12 \pm 1.66^{b}$ & $53.65 \pm 1.68^{b}$ & $51.88 \pm 1.59^{b}$ & $43.40 \pm 1.69^{a}$ & $55.39 \pm 2.95^{b}$ & $74.30 \pm 5.94^{d}$ & $67.12 \pm 2.67 \mathrm{c}$ & \\
\hline WG $(g)^{1}$ & $30.73 \pm 0.67 \mathrm{a}$ & $41.42 \pm 6.66^{b}$ & $39.85 \pm 1.66^{b}$ & b $42.38 \pm 1.72^{b}$ & $40.57 \pm 1.63^{b}$ & $1.73 \pm 2.47^{a}$ & $4.52 \pm 3.92^{b}$ & $23.43 \pm 5.99 d$ & $16.58 \pm 2.46^{c}$ & $27.09 \pm 2.34 \mathrm{e}$ \\
\hline $\operatorname{SGR}(\%)^{2}$ & $2.27 \pm 0.03^{a}$ & $2.64 \pm 0.22^{b}$ & $2.60 \pm 0.06^{b}$ & $2.69 \pm 0.06^{b}$ & $2.63 \pm 0.06^{b}$ & $0.14 \pm 0.20^{a}$ & $0.30 \pm 0.28 b$ & $1.33 \pm 0.31^{d}$ & $1.01 \pm 0.12^{c}$ & $1.52 \pm 0.09 \mathrm{e}$ \\
\hline $\mathrm{FCR}^{3}$ & $1.61 \pm 0.04^{a}$ & $1.35 \pm 0.11^{b}$ & $1.40 \pm 0.08^{b}$ & $1.29 \pm 0.05^{b}$ & $1.35 \pm 0.02^{b}$ & $2.05 \pm 0.45^{d}$ & $1.75 \pm 0.36^{c}$ & $1.08 \pm 0.69 \mathrm{a}$ & $1.18 \pm 0.56^{b}$ & $1.02 \pm 0.57 a$ \\
\hline HSI $(\%)^{4}$ & $2.08 \pm 0.08^{a}$ & $1.47 \pm 0.01^{b}$ & $1.39 \pm 0.08^{b}$ & $1.42 \pm 0.01^{b}$ & $1.56 \pm 0.02^{\mathrm{b}}$ & $1.45 \pm 0.05^{b}$ & $1.55 \pm 0.11 \mathrm{c}$ & $1.14 \pm 0.07^{a}$ & $1.54 \pm 0.17 \mathrm{c}$ & $1.51 \pm 0.09 c$ \\
\hline VSI $(\%)^{5}$ & $12.15 \pm 1.22^{\mathrm{a}}$ & $12.94 \pm 0.49 \mathrm{a}$ & $12.68 \pm 1.41^{a}$ & $13.16 \pm 0.15^{a}$ & $12.87 \pm 0.08^{a}$ & $11.22 \pm 0.63^{a}$ & $11.29 \pm 0.72^{\mathrm{a}}$ & $13.53 \pm 0.99 b$ & $13.07 \pm 0.06^{b}$ & $11.15 \pm 0.93$ \\
\hline Survival (\%) & $94.12 \pm 3.39 a$ & $100^{b}$ & $100^{b}$ & $100^{\mathrm{b}}$ & $100^{b}$ & $54.17 \pm 1.39 \mathrm{a}$ & $100^{b}$ & $100^{b}$ & $100^{\mathrm{b}}$ & $100^{\mathrm{b}}$ \\
\hline
\end{tabular}
Data are the mean values of three replicate \pm SD. Means with different superscript letter in a row are significantly different $(p>0.05)$.
${ }^{1}$ Weight gain $(W G, g)=$ Final body weight - initial body weight. ${ }^{2}$ Specific growth rate $(S G R)=\left[(\right.$ In final wet weight - In initial wet weight)/days $] \times 100 .{ }^{3}$ Feed conversion rate $(F C R)=$ total feed intake/weight gain.

\section{Proximate composition of fillets}

Fish fillet proximate compositions were significantly influenced by dietary groups at optimum temperature (Table 4, $p<0.05)$. However, except for ash, moisture, crude protein and lipid were not different in the experimental groups compared to the initial samples. In addition, the moisture content in the groups fed by FO based diet was higher than in the other groups $(p<0.05)$.

Except for crude protein, differences in moisture, ash and crude lipid contents were determined in fish fed by FO diet at upper-optimum temperature (Table $4, p>0.05$ ). The lowest moisture and fat were detected in the SFO group. The highest protein and fat and the lowest ash were in the $\mathrm{CO}$ group. High moisture and low crude lipid were determined in the FO group.
Table 4. Chemical composition of fillet of rainbow trout fingerlings for different phases of the experiment

\begin{tabular}{|c|c|c|c|c|}
\hline & Moisture (\%) & Protein (\%) & Lipid (\%) & Ash (\%) \\
\hline \multicolumn{5}{|c|}{ Optimum Temperature $\left(17^{\circ} \mathrm{C}\right)$} \\
\hline Initial & $81.65 \pm 0.44^{a}$ & $15.81 \pm 0.18^{\mathrm{a}}$ & $2.66 \pm 0.01^{a}$ & $2.64 \pm 0.09^{\mathrm{a}}$ \\
\hline FO(Control) & $76.50 \pm 0.07^{b}$ & $18.54 \pm 0.13^{b}$ & $5.73 \pm 0.68^{b}$ & $2.58 \pm 0.05^{\mathrm{a}}$ \\
\hline CANO & $75.49 \pm 0.10^{C}$ & $19.26 \pm 0.02^{b}$ & $6.70 \pm 0.23^{b}$ & $2.22 \pm 0.13^{\mathrm{a}}$ \\
\hline SFO & $75.31 \pm 0.05^{c}$ & $19.14 \pm 0.14^{b}$ & $5.61 \pm 0.37^{b}$ & $2.27 \pm 0.19^{\mathrm{a}}$ \\
\hline co & $74.72 \pm 0.03^{C}$ & $18.76 \pm 0.22^{b}$ & $5.59 \pm 0.84^{b}$ & $2.16 \pm 0.15^{\mathrm{a}}$ \\
\hline PNO & $75.33 \pm 0.05^{C}$ & $18.74 \pm 0.15^{b}$ & $5.96 \pm 0.70^{b}$ & $2.18 \pm 0.14^{\mathrm{a}}$ \\
\hline \multicolumn{5}{|c|}{ Upper-Optimum Temperature $\left(19^{\circ} \mathrm{C}\right)$} \\
\hline FO(Control) & $77.28 \pm 0.06^{d}$ & $20.56 \pm 0.13^{\mathrm{a}}$ & $3.86 \pm 0.47^{\mathrm{a}}$ & $2.35 \pm 0.41^{b}$ \\
\hline CANO & $76.82 \pm 0.02^{\mathrm{C}}$ & $20.53 \pm 0.48^{a}$ & $4.76 \pm 0.23^{b c}$ & $3.19 \pm 0.44^{\mathrm{a}}$ \\
\hline SFO & $74.59 \pm 0.03^{a}$ & $20.74 \pm 0.39^{\mathrm{a}}$ & $4.47 \pm 0.25^{b}$ & $2.83 \pm 0.06^{\mathrm{a}}$ \\
\hline CO & $76.60 \pm 0.04^{c}$ & $21.10 \pm 0.30^{\mathrm{a}}$ & $5.47 \pm 0.03^{\mathrm{C}}$ & $2.33 \pm 0.91^{b}$ \\
\hline PNO & $75.45 \pm 0.06^{b}$ & $20.32 \pm 0.26^{a}$ & $4.87 \pm 0.11^{b c}$ & $2.59 \pm 0.31^{b}$ \\
\hline
\end{tabular}




\section{Fatty acid composition of the fillets}

Total lipid and fatty acid profiles showed the effects of the respective dietary treatments (Table 5 ). The levels of SFA were significantly increased $(p<0.05)$ in fish fed by VO based diets, whereas the groups fed by FO based diet had the lowest content $(28.88 \%)$ at optimum temperature. Among the FA classes, fillet contents of monoenes were reduced in the groups fed by CANO, SFO and CO based diets, except groups fed by PNO based diet. Although the PNO based diet was the lowest MUFA content, the groups fed by PNO based diet had the highest fillet MUFA content. The highest LA increase was observed in the groups fed by SFO based diet having the lowest value of $20.92 \%(p<0.05)$. Likewise, the inclusion of vegetable oils in diet led to increased ALA content $(p<0.05)$ in all groups fed by VO based diets, apart from groups fed by SFO based diet. While EPA and DHA were significantly high $(p<0.05)$ in the groups fed by FO based diet in comparison to the other groups, ARA was increased in the groups fed by CO, SFO, PNO and CANO based diets, respectively. The groups fed by $F O$ based diet had the highest $(p<0.05) n-3$ PUFA content, EPA/DHA and $n-3 / n-6$ ratios.

LA content of the SO group decreased significantly after 4 weeks from the beginning of the upper-optimum temperature trial and reached almost the same level of the FO group at the end of the trial (Table 5). The same situation was also observed for OA content. EPA, DHA and ARA contents of fish in the growth out period were increased significantly after 4 weeks of FOFD trial. At the end of the growth-out trial period, although fish in the FO group had the highest $(p<0.05) n-3$ PUFA content, EPA/DHA and n-3/n-6 ratios, after 4 weeks of FOFD trial, $n-6$ PUFA ratios decreased and $n-3$ ratios increased. In all groups, EPA/DHA was equal.

Table 5. Fatty acid compositions of fillet of rainbow trout fingerlings fed experimental diets (\% of total fatty acids) at optimum and upperoptimum temperature

\begin{tabular}{|c|c|c|c|c|c|c|c|c|c|c|}
\hline & \multicolumn{5}{|c|}{ Optimum Temperature $\left(17^{\circ} \mathrm{C}\right)$} & \multicolumn{5}{|c|}{ Upper-Optimum Temperature $\left(19^{\circ} \mathrm{C}\right)$} \\
\hline & FO & CANO & SFO & $\mathrm{CO}$ & PNO & FO & CANO & SFO & $\mathrm{CO}$ & PNO \\
\hline C14:0 & $4.04 \pm 0.00^{a}$ & $2.93 \pm 0.91^{b}$ & $1.51 \pm 0.00^{c}$ & $1.53 \pm 0.02^{c}$ & $1.63 \pm 0.00^{c}$ & $3.54 \pm 0.05^{a}$ & $2.49 \pm 0.01 \mathrm{~cd}$ & $2.42 \pm 0.00 \mathrm{~d}$ & $2.53 \pm 0.04 \mathrm{c}$ & $2.66 \pm 0.03 b$ \\
\hline C15:0 & $20 \pm 0.00^{a}$ & $0.74 \pm 0.38^{b}$ & $0.27 \pm 0.00^{c}$ & $0.27 \pm 0.01 c$ & $0.30 \pm 0.01 \mathrm{bc}$ & $04 \pm 0.01^{a}$ & $0.62 \pm 0.01 \mathrm{c}$ & $0.60 \pm 0.01 \mathrm{c}$ & $0.66 \pm 0.02 b$ & $0.68 \pm 0.02 b$ \\
\hline $\mathrm{C} 16: 0$ & $12.18 \pm 0.06^{b}$ & $12.84 \pm 0.20^{a}$ & $10.10 \pm 0.00 c$ & $12.06 \pm 0.21^{b}$ & $11.90 \pm 0.07^{b}$ & $3.14 \pm 0.23^{a}$ & $11.38 \pm 0.01 \mathrm{~d}$ & $11.58 \pm 0.00 \mathrm{~cd}$ & $11.94 \pm 0.15 b d$ & $12.26 \pm 0.18 b$ \\
\hline & $1.38 \pm 0.00^{\mathrm{a}}$ & $0.79 \pm 0.47^{\mathrm{b}}$ & & $0.23 \pm 0.00^{b c}$ & $0.28 \pm 0.00^{\mathrm{bc}}$ & $.20 \pm 0.03 a$ & & $0.58 \pm 0.00 d$ & $0.73 \pm 0.02 b$ & $0.71 \pm 0.01 b c$ \\
\hline C18:0 & $6.46 \pm 0.03^{b}$ & $7.85 \pm 0.77^{a}$ & $7.80 \pm 0.03^{a}$ & $7.74 \pm 0.15^{\mathrm{a}}$ & $7.97 \pm 0.04 \mathrm{a}$ & $7.36 \pm 0.13 b$ & & $7.78 \pm 0.02 a$ & & $7.92 \pm 0.09 a$ \\
\hline C20:0 & $0.92 \pm 0.01^{c}$ & $1.81 \pm 0.94$ bc & $3.58 \pm 0.01^{a}$ & $3.41 \pm 0.10^{a}$ & $2.61 \pm 0.02^{\mathrm{ab}}$ & $0.84 \pm 0.02 e$ & $1.75 \pm 0.01 d$ & $2.89 \pm 0.00 \mathrm{a}$ & $2.79 \pm 0.06 \mathrm{~b}$ & $2.00 \pm 0.03 c$ \\
\hline $\mathrm{C} 22: 0$ & $2.05 \pm 0.01^{c}$ & $2.45 \pm 0.51 \mathrm{bc}$ & $3.26 \pm 0.00^{a}$ & $3.29 \pm 0.05^{a}$ & $2.98 \pm 0.02^{\mathrm{ab}}$ & $2.20 \pm 0.03 d$ & $2.94 \pm 0.02 b$ & $3.16 \pm 0.01 \mathrm{a}$ & $3.19 \pm 0.06 a$ & $2.80 \pm 0.04 \mathrm{c}$ \\
\hline C24:0 & $0.66 \pm 0.01^{a}$ & $0.36 \pm 0.26^{\mathrm{ab}}$ & $0.07 \pm 0.01^{b}$ & $0.08 \pm 0.05^{b}$ & $0.08 \pm 0.01^{b}$ & $0.54 \pm 0.01 a$ & $0.28 \pm 0.01 \mathrm{c}$ & $0.26 \pm 0.01 \mathrm{c}$ & $0.31 \pm 0.02 b$ & $0.32 \pm 0.01 b$ \\
\hline$\Sigma S F A$ & $28.88 \pm 0.11^{b}$ & $29.75 \pm 0.41^{a}$ & $26.79 \pm 0.004^{d}$ & $28.59 \pm 0.01^{b}$ & $27.73 \pm 0.14 c$ & $29.85 \pm 0.51 a$ & $27.23 \pm 0.09 b$ & $29.25 \pm 0.04 a$ & $29.25 \pm 0.49 a$ & $29.32 \pm 0.39 a$ \\
\hline C14:1 & $0.42 \pm 0.01 a$ & $0.24 \pm 0.15^{\mathrm{ab}}$ & $0.07 \pm 0.00^{\mathrm{b}}$ & $0.07 \pm 0.54^{b}$ & $0.08 \pm 0.00^{b}$ & $0.35 \pm 0.00 \mathrm{a}$ & $0.19 \pm 0.01 d$ & $0.17 \pm 0.00 \mathrm{e}$ & $0.20 \pm 0.00 \mathrm{c}$ & $0.22 \pm 0.01 b$ \\
\hline & $27 \pm 0.04^{a}$ & $0.12 \pm 0.07^{b}$ & & $0.04 \pm 0.00^{\mathrm{b}}$ & $4 \pm 0.00^{b}$ & $0.21 \pm 0.04 a$ & & $\pm 0.01 b c$ & $.02 b c$ & $0.18 \pm 0.00 \mathrm{ab}$ \\
\hline & & $4.29 \pm 0.96^{b}$ & & $2.49 \pm 0.00^{c}$ & $2.74 \pm 0.00^{c}$ & $15 \pm 0.10 \mathrm{a}$ & & & & $4.02 \pm 0.06 b$ \\
\hline & & $55 \pm 0.21^{b}$ & & $\pm 0.04 c$ & & & & & & \\
\hline & $3.35 \pm 0.04 c$ & $10.46 \pm 2.30^{d}$ & $18.32 \pm 0.04^{b}$ & $18.57 \pm 0.00^{\mathrm{b}}$ & $22.91 \pm 0.10^{a}$ & $1.54 \pm 1.43 c$ & $0.04 a$ & $32 \pm 0.08 b$ & $\pm 0.20 \mathrm{~b}$ & $8.60 \pm 1.35 a$ \\
\hline & $2.68 \pm 0.06^{\mathrm{ab}}$ & $3.23 \pm 0.61^{a}$ & $1.85 \pm 0.01^{c}$ & $4 \pm 0.32^{c}$ & $2.25 \pm .015^{b c}$ & $2.61 \pm 0.08 \mathrm{ab}$ & $2.72 \pm 0.17 a$ & $\pm 0.00 \mathrm{ab}$ & $42 \pm 0.00 \mathrm{ab}$ & $26 \pm 0.25 b$ \\
\hline C20:1 & $2.67 \pm 0.00^{\mathrm{abc}}$ & $3.43 \pm 0.90^{\mathrm{ab}}$ & $2.24 \pm 0.01^{c}$ & \pm 0.04 bc & $3.75 \pm 0.00^{a}$ & $.48 \pm 0.03 c$ & $2.96 \pm$ & $3 \pm 0.00 \mathrm{~d}$ & $2.22 \pm 0.03 e$ & $3.24 \pm 0.04 a$ \\
\hline & $7 \pm 0.01^{a}$ & $2.12 \pm 0.39 b$ & & $\pm 0.04 c$ & $\pm 0.01^{c}$ & $\pm 0.02 \mathrm{a}$ & & & $.04 \mathrm{c}$ & $\pm 0.03 b c$ \\
\hline & $1.48 \pm 0.01^{c}$ & $1.73 \pm 0.29 c$ & & $3.02 \pm 0.01^{a}$ & $3 \pm 0.03^{b}$ & $1.52 \pm 0.02 d$ & & $\pm 0.00 \mathrm{a}$ & $=0.04 a$ & $\pm 0.03 b$ \\
\hline & $29.96 \pm 0.02^{b}$ & $26.16 \pm 2.27 c$ & $29.14 \pm 0.02^{b}$ & $9 \pm 0.05^{b}$ & $89 \pm 0.20^{a}$ & $26.77 \pm 1.13 c$ & $09 a$ & $5 \pm 0.06 b$ & $\pm 0.37 b c$ & $32.93 \pm 0.94 a$ \\
\hline & $11.50 \pm 0.01^{c}$ & $13.82 \pm 2.82^{c}$ & & $17.80 \pm 0.49 \mathrm{~b}$ & $14.63 \pm 0.01 \mathrm{bc}$ & $10.70 \pm 0.17 b$ & $12.12 \pm 0.05 b$ & $15.47 \pm 0.14 a$ & $\pm 1.30 \mathrm{a}$ & $11.89 \pm 0.23 b$ \\
\hline & $0.65 \pm 0.00^{a}$ & $0.15 \pm 0.04^{b}$ & & $0.12 \pm 0.00^{b}$ & $15 \pm 0.00^{b}$ & $0.10 \pm 0.00 \mathrm{e}$ & & $0.29 \pm 0.00 d$ & & $\pm 0.00 a$ \\
\hline & $08 \pm 0.01 \mathrm{bc}$ & $1.17 \pm 0.17^{b}$ & & $93 \pm 0.01^{c}$ & $53 \pm 0.01^{a}$ & $0.96 \pm 0.01 \mathrm{c}$ & & $0.83 \pm 0.00 \mathrm{e}$ & $0.03 d$ & $1 \pm 0.02 \mathrm{a}$ \\
\hline & $\pm 0.01 \mathrm{ab}$ & $5.08 \pm 1.41^{a}$ & $00^{c}$ & $\pm 0.02^{b c}$ & $0.01 \mathrm{bc}$ & $.05 b$ & $4.27 \pm$ & $0.01 \mathrm{~d}$ & $.02 c$ & $\pm 0.04 \mathrm{c}$ \\
\hline & $2.28 \pm 0.01^{c}$ & $2.72 \pm 0.57^{b c}$ & $3.31 \pm 0.00^{\mathrm{ab}}$ & $3.73 \pm 0.05^{a}$ & $3.05 \pm 0.00^{\mathrm{ab}}$ & $1.84 \pm 0.02 \mathrm{e}$ & $2.33 \pm$ & $1 \pm 0.00 \mathrm{a}$ & $.06 \mathrm{~b}$ & $2.51 \pm 0.03 c$ \\
\hline$: 3 n-6$ & $1.16 \pm 0.01^{c}$ & $2.11 \pm 1.03^{b c}$ & $1 \pm 0.00^{a}$ & $3.80 \pm 0.06^{a}$ & $3.16 \pm 0.00^{\mathrm{ab}}$ & $1.02 \pm 0.02 \mathrm{e}$ & $2.13 \pm c$ & & & $2.32 \pm 0.03 c$ \\
\hline & $2.05 \pm 0.01^{b}$ & $2.40 \pm 0.46^{b}$ & & $3.29 \pm 0.05^{a}$ & $2.98 \pm 0.02^{a}$ & $2.20 \pm 0.03 d$ & $2.94 \pm 0.02 b$ & $\pm 0.01 \mathrm{a}$ & $6 a$ & $2.80 \pm 0.04 \mathrm{c}$ \\
\hline$C 20: 5 n-3$ & $5.97 \pm 0.17 \mathrm{a}$ & $3.98 \pm 1.65^{b}$ & $0.98 \pm 0.01^{c}$ & $1.08 \pm 0.02^{c}$ & $1.42 \pm 0.02^{c}$ & $5.71 \pm 0.09 a$ & $3.91 \pm 0.01 b$ & $3.35 \pm 0.01 d$ & $3 \pm 0.10 c$ & $3.57 \pm 0.04 c$ \\
\hline C22:2 & $0.19 \pm 0.00^{a}$ & $0.15 \pm 0.00^{b}$ & $0.12 \pm 0.01 \mathrm{c}$ & $0.12 \pm 0.01^{c}$ & $0.11 \pm 0.00^{c}$ & $0.14 \pm 0.02 \mathrm{ab}$ & $0.13 \pm 0.01 b$ & $0.15 \pm 0.00 \mathrm{a}$ & $0.14 \pm 0.00 \mathrm{ab}$ & $0.14 \pm 0.00 \mathrm{ab}$ \\
\hline$C 22: 6 n-3$ & $11.95 \pm 0.01^{a}$ & $12.17 \pm 3.07^{a}$ & $4.77 \pm 0.02^{b}$ & $5.95 \pm 0.08^{b}$ & $6.68 \pm 0.02^{b}$ & $16.86 \pm 0.21 a$ & $11.61 \pm 0.01 b$ & $9.89 \pm 0.02 d$ & $10.54 \pm 0.12 c$ & $9.86 \pm 0.12 d$ \\
\hline & $40.70 \pm 0.13^{b}$ & $43.73 \pm 1.79 \mathrm{a}$ & $43.68 \pm 0.05^{a}$ & $39.25 \pm 0.65^{b}$ & $35.94 \pm 0.04 \mathrm{c}$ & $43.02 \pm 0.61 a$ & $40.76 \pm 0.00 \mathrm{~b}$ & $41.61 \pm 0.11 \mathrm{ab}$ & $42.56 \pm 0.86 a$ & $37.35 \pm 0.55 c$ \\
\hline 乏n-3PUFA & $19.00 \pm 0.17^{a}$ & $17.31 \pm 5.54^{a}$ & & $7.95 \pm 0.10^{b}$ & $9.62 \pm 0.02^{\mathrm{b}}$ & $23.53 \pm 0.31 a$ & $16.55 \pm 0.01 b$ & $14.07 \pm 0.03 d$ & $14.94 \pm 0.24 c$ & $15.33 \pm 0.42 c$ \\
\hline En-6PUFA & $19.23 \pm 0.03 c$ & $23.56 \pm 5.76 \mathrm{bc}$ & $33.84 \pm 0.04^{a}$ & $27.46 \pm 0.48^{\mathrm{ab}}$ & $23.16 \pm 0.02^{b c}$ & $19.23 \pm 0.03^{c}$ & $23.56 \pm 5.76^{b c}$ & $33.84 \pm 0.04^{a}$ & $27.46 \pm 0.48^{a b}$ & $23.16 \pm 0.02^{b c}$ \\
\hline$n-3 / n-6$ & $0.99 \pm 0.01^{a}$ & $0.83 \pm 0.40^{\mathrm{ab}}$ & $0.19 \pm 0.00^{c}$ & $0.29 \pm 0.00 c$ & $0.42 \pm 0.00 \mathrm{bc}$ & $0.99 \pm 0.01^{a}$ & $0.83 \pm 0.40^{a b}$ & $0.19 \pm 0.00 c$ & $0.29 \pm 0.00 c$ & $0.42 \pm 0.00 \mathrm{bc}$ \\
\hline EPA/DHA & $0.50 \pm 0.01^{a}$ & $0.31 \pm 0.06^{b}$ & $0.20 \pm 0.00 c$ & $0.18 \pm 0.00 c$ & $0.21 \pm 0.00 \mathrm{c}$ & $0.50 \pm 0.01^{a}$ & $0.31 \pm 0.06^{b}$ & $0.20 \pm 0.00 c$ & $0.18 \pm 0.00 c$ & $0.21 \pm 0.00^{c}$ \\
\hline
\end{tabular}

作 PUFA: Polyunsaturated fatty acids, DHA: Docosahexaenoic acid, EPA: Eicosapentaenoic acid 

optimum -and high temperature conditions

\section{The nutritional quality indices of the fillet lipids for different phases}

At the end of the optimum temperature trial, the IA, IT, $\mathrm{FLQ}$ and $\mathrm{h} / \mathrm{H}$ values decreased in the groups fed with vegetable oil diets compared to the control group. Differences were determined between groups $(p<0.05)$. At the end of the upper-optimum temperature trial, it was determined that the nutritional quality indexes increased in the groups fed with vegetable oil diets (Table 6).

Table 6. The nutritional quality indices of the fillet lipids of rainbow trout fingerlings for different phases of the experiment

\begin{tabular}{|c|c|c|c|c|}
\hline & IA & IT & FLQ & $\mathrm{h} / \mathrm{H}$ \\
\hline \multicolumn{5}{|c|}{ Optimum Temperature $\left(17^{\circ} \mathrm{C}\right)$} \\
\hline FO(Control) & $0.42 \pm 0.00^{a}$ & $0.27 \pm 0.00^{c}$ & $18.00 \pm 0.08^{a}$ & $2.83 \pm 0.00^{d}$ \\
\hline CANO & $0.37 \pm 0.03^{a}$ & $0.31 \pm 0.03^{b c}$ & $16.20 \pm 2.72^{a}$ & $2.79 \pm 0.06^{d}$ \\
\hline SFO & $0.23 \pm 0.00^{b}$ & $0.38 \pm 0.00^{a}$ & $5.76 \pm 0.01^{b}$ & $4.56 \pm 0.00^{a}$ \\
\hline CO & $0.28 \pm 0.00^{\mathrm{b}}$ & $0.40 \pm 0.00^{\mathrm{a}}$ & $7.18 \pm 0.01^{b}$ & $3.50 \pm 0.00^{c}$ \\
\hline PNO & $0.27 \pm 0.00^{b}$ & $0.37 \pm 0.00^{\mathrm{ab}}$ & $8.13 \pm 0.02^{b}$ & $3.71 \pm 0.01^{b}$ \\
\hline \multicolumn{5}{|c|}{ Upper-optimum Temperature $\left(19^{\circ} \mathrm{C}\right)$} \\
\hline $\mathrm{FO}$ (Control) & $0.40 \pm 0.00 \mathrm{a}$ & $0.26 \pm 0.00^{c}$ & $22.65 \pm 0.17^{a}$ & $2.88 \pm 0.06^{c}$ \\
\hline CANO & $0.31 \pm 0.00^{d}$ & $0.27 \pm 0.00^{b}$ & $15.57 \pm 0.02^{b}$ & $3.57 \pm 0.00^{a}$ \\
\hline SFO & $0.32 \pm 0.00^{\mathrm{cd}}$ & $0.31 \pm 0.00^{a}$ & $13.29 \pm 0.02^{d}$ & $3.43 \pm 0.00^{\mathrm{ab}}$ \\
\hline CO & $0.33 \pm 0.00^{\mathrm{bc}}$ & $0.30 \pm 0.00 \mathrm{a}$ & $14.13 \pm 0.13^{c}$ & $3.34 \pm 0.06^{b}$ \\
\hline PNO & $0.34 \pm 0.01^{b}$ & $0.31 \pm 0.00^{a}$ & $13.48 \pm 0.09^{d}$ & $3.22 \pm 0.06^{b}$ \\
\hline
\end{tabular}

Data are mean $\pm S D$. Means with different superscript letter in a column are significantly different $(p>0.05)$

\section{DISCUSSION}

Fish oil has been widely used as the main lipid source of the $n-3$ polyunsaturated fatty acids ( $n-3$ PUFA) in farmed fish feed. Fish oil production has decreased in recent years due to the decrease in fish stocks. These increases feed costs considerably. Thus, the current trend is finding suitable alternatives to fish oils in aquafeeds, without compromising overall growth performance and final nutritional quality of farmed fish. In this case, the best alternative could be vegetable oil. However, the alternative vegetable oil is poor sources for n-3 HUFAs. One way to improve the nutritional quality of farmed fish fed by alternative vegetable oil may be to use finishing feeds containing fish oil before harvesting. Firstly, the aim of this study was to determine the effect of feeding with vegetable oil-based diets for 9 weeks at optimum temperature on the growth, feed utilization and fatty acid profile of rainbow trout. Secondly, by continuing the study for another 4 weeks, all groups at upper-optimum temperature were re-fed with only fish oil-based diet (control), and at the end of this period, it was aimed to determine the gradual recovery of fatty acid profiles and nutritional quality of rainbow trout and especially its effect on survival rate.
A number of earlier studies have utilized vegetable oil in feed formulations for salmonids at a replacement level of up to $100 \%$, and no negative effect in growth rates and feed conversion have been observed (Torstensen et al. 2000; Caballero et al. 2002; Fonseca-Madrigal et al., 2005; Yildiz et al. 2015; Yıldız et al. 2018; Yu et al. 2018). Similar results were determined in the present study. However, after feding the fish by VO based diets in the growth-out period, fish weights in groups fed by FO based diet was less than the other experimental groups, despite final fish weights of the all trial groups increased in 9 weeks at the top optimal temperature. In parallel with this situation, the lowest WG, FCR, SGR, survival and highest HSI were detected in the groups fed by FO based diet. Similar results were also reported by Babalola and Apata (2012). In their study using different vegetable oil (soybean, palm, coconut, groundnut and melon seed oil) in Heterobranchus longifilis fingerling diets, although survival, feed intake and HSI were similar for all experimental groups, WG, SGR and FCR of fish fed by soybean oil based diet decreased significantly. As a result, it was reported that the usage of different vegetable oils in the diets were created no negative effect on ther growth composition of Heterobranchus longifilis fingerlings. VSI values of the present study were equal for both groups fed by FO and VO based diets in the growth-out period. Yildiz et al. (2015) reported that the VSI value of rainbow trout fed by diet including sunflower oil was lower than the those of fish fed by diets including other vegetable oil (sesame and linseed oil).

In the optimum temperature trial, while the survival rate decreased in the groups fed by FO based diet, it was not changed in the other groups. After previously fed by VO based diets, $\mathrm{HSI}, \mathrm{VSI}$ and growth performance of fish fed by FO diet for four weeks at upper-optimum temperature were different among the all trial. The decrease in the survival rate determined in the optimum temperature trial continued in the upper-optimum temperature trial. While the survival rates of all groups (CANO, SFO, CO, PNO groups) in the upperoptimum temperature trial were $100 \%$, this rate decreased to $54.17 \pm 1.39 \%$ in the groups fed by FO based diet. In the present study, feeding with vegetable oil diets at top and upper optimal temperatures was most effective at the survival rate. This case could be originated from the antioxidant property of the vegetable oils. It was proved that vitamin $E$, one of the strongest natural antioxidants (Altıner et al., 2017), has an immune-enhancing effect compared to a synthetic antioxidant (McDowell et al. 1996). Canola, sunflower, corn and peanut oils used in the present study are also rich in vitamin $E$ (İşler, 2018). Also, It was reported that the presence of low vitamin $E$ in the fish diets decreased the resistance to stress of the fish under acute and chronic stress and increased mortality (Montero et al., 2001).

After the optimum temperature trial period, fatty acid profiles of diets containing VO were reflected the FA profiles of fish fillet, except for groups fed by CANO based diet which was characterized by high LA and OA and low n-3 PUFA 
contents. In the present study, OA, LA and n-3 PUFA contents in the groups fed by CANO based diet were almost same as those in the groups fed by FO based diet. Several studies showed that salmonids respond differently to distinct VO, making it difficult to make direct comparison among studies. Bell et al., (2003) reported that fillet fatty acid profile showed significant increases in OA and LA as well as ALA with the addition of rapeseed oil in the Atlantic salmon (Salmo salar) diets. Bell et al. (2001) reported that the inclusion of RO in levels exceeding of $50 \%$ may result in significant reductions in $(n-3) /(n-6)$ PUFA ratio and the EPA and DHA concentrations, but, this does not prevent higher rapeseed oil levels from being used in dietary formulations for Atlantic salmon, such diets could be brought back to "normal" values of 18: 2 ( $n-6)$, EPA and DHA concentrations by feeding with fish oil diets at a convenient time before marketing. Yildiz et al. (2015) reported that $100 \%$ replacement of fish oil with vegetable oil (sunflower, sesame and linseed oil) resulted in decrease of total n-3 PUFA levels and increased levels of $n-6$ PUFA in both fillet and liver, except for linseed oil groups in rainbow trout. They also indicated increased levels of $n-6$ and $L A$ with inclusion of sunflower oil whereas ALA increased with inclusion of linseed oil. The reduced accumulation of dietary monoenes and LA coupled with good growth, survival and feed utilization in their study were capable of meeting essential fatty acid (EFA) requirements of rainbow trout by the use of monoenes and LA. This stiuation was also reported that residual fish oil in the fishmeal in the diets containing VO could be enough to provide essential HUFAs for normal growth and development of rainbow trout (Yıldız et al., 2018).

In the present study, DHA and ARA concentrations in fish fillet were higher than the dietary concentrations. This case was also detected by Caballero et al. (2002) for rainbow trout. The simlar results were also reported for DHA levels in European sea bass (Montero et al., 2005; Mourente and Bell, 2006), gilthead sea bream (Sparus aurata L.) (Menoya et al., 2004) and turbot (Psetta maxima) (Regost et al., 2003). Specific fatty acids were selectively retained in the fish fillet; especially DHA and ARA concentrations were higher than the dietary concentrations (Fountoulaki et al., 2009).

At the end of the upper-optimum temperature trial, the fatty acid composition of groups fed by CANO based diet was closer to the the fatty acid composition of the groups fed by FO based diet. The fatty acid composition of the other groups increased almost to the doubles. Similar results declared by Yıldız et al. (2018) and Codabaccus et al. (2013) for rainbow trout and by $\mathrm{Ng}$ et al.,o (2013) for red hybrid tilapia (Oreochromis sp.), Fountoulaki et al. (2009) for gilthead sea bream and Bell et al. (2003) for Atlantic salmon.

Total substitution of dietary FO with vegetable oils (CANO, SFO, CO and PNO) caused a decline in the fillet PUFAs (EPA and DHA) in the present study. This is compatibles previous studies (Caballero et al., 2002; Bell et al., 2004; Thanuthong et al., 2011; Codabaccus et al., 2013; Yıldız et al., 2018). At the end of the growth-out trial, DHA and
EPA were significantly lower. PUFA retention in the present study followed the pattern; DHA>EPA. DHA showed higher retention than EPA in fish fed by VO based diets. At the end of the trial, an increase in DHA and EPA in the fillets was detected in all groups. The present study showed that 4 weeks at upper-optimum temperature for the restoration of ARA, EPA and DHA was sufficient, especially in the groups fed by CANO based diet. Returning fish previously fed by diet including $100 \%$ vegetable oil to fish oil diet for a period before harvest allowed restoration of flesh (n-3) HUFA concentrations up to $80 \%$ comparing with fish fed by FO throughout the seawater phase, although 18:2(n-6) remained significantly higher (Sonu, 2018). It was declared that the fatty acid composition of the fish fillets was restored applying a FOFD strategy for 16 weeks after feeding with vegetable oil diets (canola and linseed oils) for 25 weeks in murray cod (Maccullochella peelii peelii, Mitchell) (Turchini at al., 2006). However, studies with marine fish emphasized that ARA and DHA but not EPA could be restored by longer FOFD trials (trials $\geq 5$ months) (Yıldız et al., 2018). It was reported that EPA levels were not restored even after 104 days of FOFD application in gilthead sea bream (Izquierdo et al., 2005), 150 days of FOFD application in European sea bass (Montero et al., 2005), 56 days of FOFD application in turbot (Regost et al., 2003). However, Fountoulaki et al. (2009) concluded that re-feeding gilthead sea bream previously fed by VO based diets with a FOFD for 120 days was not adequate for restoration of DHA, ARA and EPA. This stuation suggests that rainbow trout has the ability to convert fatty acids more quickly.

Several indicators have been described to determine the nutritional quality of food lipids in human consumption. In the present study, to identify the nutritional quality of fillet lipids, the IA, IT, FLQ and $h / H$ levels were measured in fillets. In accordance with nutritional recommendations, it was reported that lipids with lower indices of atherogenicity (IA) and thrombogenicity (IT) can hinder the aggregation of platelets and reduce the levels of esterified FA, cholesterol and phospholipids, thereby preventing the appearance of microand macro-coronary diseases (Turan et al., 2007). That's why IA and IT indexes should be low to prevent cardiovascular diseases related to lipid intake (Ulbricht and Southgate, 1991). However, the high value of $\mathrm{h} / \mathrm{H}$ ratio represents highquality lipids. In this study, IA and IT indices were measured to be lower than 1.0 but high in $\mathrm{h} / \mathrm{H}$ values in all groups. Ouraji et al. (2009) and Stancheva et al. (2014) determined that higher values of IA and IT (>1.0) are detrimental to human health. Monge-Ortiz et al. (2018) reported that lower levels of IA and IT values are beneficial to human health. Flesh Lipid Quality Indice (FLQ) indicates the global dietetic quality of lipids and their potential effects on the development of coronary disease (Senso et al., 2007). Lipid quality of the flesh is directly related to EPA and DHA ratios (Dagtekin et al., 2017). A relatively high FLQ value was obtained in this study. It was reported that omega- 3 fatty acids, which are beneficial for both healthy and people suffering from 

optimum -and high temperature conditions

cardiovascular diseases, are lower the risk of arrhythmias, which can lead to sudden death (URL 2, 2016). Therefore, Yu et al. (2018) reported that consuming both the IA and IT indices together with high FLQ with low efficacy is an indication that it may help prevent the development of coronary heart disease, which is more favorable in terms of lipid quality for human consumption. In this context, it can be said that the European seabass obtained after the present study have a good nutritional quality for human consumption.

As a result, this study was conducted to investigate the growth effectiveness of rainbow trout fed by $\mathrm{VO}$ based diets at optimum temperature and then fed by FO diet at upperoptimum temperature. Optimum temperature trial results of current study suggest that it is possible to replace FO totally with $\mathrm{VO}$ in rainbow trout diets without any significant effect on growth and feed utilization. FA profile of fish fed by VO based diets reflected dietary FA composition characterized by reduced contents of ARA, EPA and DHA. Feeding by diets

\section{REFERENCES}

Altıner, A., Atalay, H. \& Bilal, T. (2017). Bir antioksidan olarak E vitamini. Balıkesir Sağılk Bilimleri Dergisi, 6(3),149-157. DOI: $10.5505 / b s b d .2017 .47450$

AOAC, (1995). Animal Feed. W. Horwitz (Ed.). Official Methods of Analysis of the Association of Analytical Chemists, 13th Edition 7, 125. USA

Babalola, T.O. \& Apata, D.F. (2012). Effects of dietary lipid source on growth, digestibility and tissue fatty acid composition of Heterobranchus longifilis fingerlings. Journal of Agriculture and Rural Development in the Tropics and Subtropics, 113(1), 1-11

Bayaz, M. (2014). Esansiyel yağlar: Antimikrobiyal, antioksidan ve antimutajenik aktiviteleri. Akademik Gıda, 12(3), 45-53

Bell, J.G., McEvoy, J., Tocher, D.R., McGhee, F., Campbell, P.J. \& Sargent, J.R. (2001). Replacement of fish oil with rapeseed oil in diets of Atlantic salmon (Salmo salar) affects tissue lipid compositions and hepatocyte fatty acid metabolism. J Nutr., 131, 1535-1543.

DOI: 10.1093/jn/131.5.1535

Bell, J.G., Tocher, D.R., Henderson, R.J., Dick, J.R. \& Cramptom, V.O (2003). Altered fatty acid compositions in Atlantic salmon (Salmo salar) fed diets containing linseed and rapeseed oils can be partially restored by a subsequent fish oil finishing diet. J Nutr., 133, 2793-2801. DOI: 10.1093/jn/133.9.2793

Bell, J.G., Henderson, R.J., Tocher, D.R. \& Sargent, J.R. (2004). Replacement of dietary fish oil with increasing levels of linseed oil: modification of flesh fatty acid compositions in Atlantic salmon (Salmo salar) using a fish oil finishing diet. Lipids, 39(3), 223-232. DOI: 10.1007/s11745-004-1223-5

Caballero, M.J., Obach, A., Roselund, G., Montero, D., Gisvold, M. \& Izquierdo, M.S. (2002). Impact of different dietary lipid sources on growth, lipid digestibility, tissue fatty acid composition and histology of rainbow trout, Oncorhynchus mykiss. Aquaculture, 214, 253-271. DOI: 10.1016/S0044-8486(01)00852-3

Codabaccus, M.B., Ng, W.-K., Nichols, P.D. \& Carter, C.G. (2013). Restoration of EPA and DHA in rainbow trout (Oncorhynchus mykiss) using a finishing fish oil diet at two different water temperatures. Food Chemistry, 141, 236-244. DOI: 10.1016/j.foodchem.2013.02.032

Dagtekin, B.B.G., Misir, G.B., Kutlu, S. \& Basturk, O. (2017). Comparison of biochemical, fatty acids and lipid quality indexes of Prussian carp (Carassius gibelio) caught from Lake Çıldır on different seasons. Mediterranean Fisheries and Aquaculture Research, 1(1), 2-14.

Fonseca-Madrigal, J., Karalazos, V., Campbell, P.J., Bell, J.G. \& Tocher, D.R. (2005). Influence of dietary palm oil on growth, tissue fatty acid composition, and fatty acid metabolism in liver and intestine in rainbow trout (Oncorhynchus mykiss). Aquac. Nutr., 11, 241-250. DOI: 10.1111/j.1365-2095.2005.00346.x including different VO during nine weeks of optimum temperature period followed by a FO diet for four weeks at upper-optimum temperature was sufficient for a large restoration \% values of EPA and DHA in rainbow trout fillet. Although a large restoration of EPA and DHA was achieved for fish previously fed by VO based diets at both optimum and upper-optimum temperatures, the $n-3 / n-6$ ratios were not fully restored in the all groups of upper-optimum temperature trial. In spite of this, implementing such a feeding strategy to rainbow trout culture will probably offer the best balance between feed cost-effectiveness and fillet quality.

\section{ACKNOWLEDGEMENTS}

This study was conducted at the Research and Application Center of the Aquaculture and Fisheries Faculty of Sinop University, Turkey. We extend our gratefulness to Osman PARLAK, the Owner of the Kuzey Su Urunleri fish farm, for providing us the fish.

Gabor, E.F., Ichim, O. \& Şuteu, M. (2012). Phyto-additives in rainbow trout (Oncorhynchus mykiss) nutrition. Biharean Biologist, 6(2), 134-139

Fountoulaki, E., Vasilaki, A., Hurtado, R., Grigorakis, K., Karacostas, I., Nengas, I., Rigos, G., Kotzamanis, Y., Venou, B. \& Alexis, M.N. (2009). Fish oil substitution by vegetable oils in commercial diets for gilthead sea bream (Sparus aurata L.); effects on growth performance, flesh quality and fillet fatty acid profile: Recovery of fatty acid profiles by a fish oil finishing diet under fluctuating water temperatures. Aquaculture, 289, 317-326. DOI: 10.1016/j.aquaculture.2009.01.023

Hanson, S.W.F. \& Olley, J. (1963). Application of the Bligh and Dyer method of lipid extraction to tissue homogenates. Biochem J., 89, 101-102

Izquierdo, M.S., Montero, D., Robaina, L., Caballero, M.J., Rosenlund, G. \& Ginés, R. (2005). Alterations in fillet fatty acid profile and flesh quality in gilthead seabream (Sparus aurata) fed vegetable oils for a long term period. Recovery of fatty acid profiles by fish oil feeding. Aquaculture, 250, 431-444. DOI: 10.1016/j.aquaculture.2004.12.001

İşler, N. (2018). Kolza (Kanola) tarımı. M.K.Ü. Ziraat Fakültesi Tarla Bitkileri Bölümü. http://www.mku.edu.tr/files/898-0e9fe1a8-1d54-4c38-8ac4cdbdccae03ad.pdf

Kocatepe, D., Erdem, M.E., Keskin, I., Köstekli, B. \& Kaya Y. (2019). Differences on lipid quality index and amino acid profiles of European Anchovy caught from different area in Turkey. Ukrainian Journal of Food Science, 7(1), 6-15. DOI: 10.24263/2310-1008-2019-7-13

McDowell, L.R., Williams, S.N., Hidiroglou, N., Njeru, C.A., Hill, G.M., Ochoa, L. \& Wilkinson, N.S. (1996). Vitamin E supplementation for the ruminant. Animal Feed Science Technology, 60, 273-296. DOI: 10.1016/0377-8401(96)00982-0

Menoyo, D., Izquierdo, M.S., Robaina, L., Cinés, R., Lopez-Bote, C.J. \& Bautista, J.M. (2004). Adaptation of lipid metabolism, tissue composition and flesh quality in gilthead sea bream (Sparus aurata) to the replacement of dietary fish oil by linseed and soyabean oils. Br. J. Nutr., 92, 41-52. DOI: 10.1079/BJN20041165

Monge-Ortiz, R., Tomas-Vidal, A., Rodriguez-Barreto, D., Martínez-Llorens, S., Perez, J., Jover Cerda, M., Lorenzo, A. (2018). Replacement of fish oil with vegetable oil blends in feeds for greater amberjack (Seriola dumerili) juveniles: effect on growth performance, feed efficiency, tissue fatty acid composition and flesh nutritional value. Aquaculture Nutrition, 24(1),605-615. DOI: 10.1111/anu.12595

Montero, D., Tort, L., Robaina, L.,Vergara, J.M. \& Izquierdo, M.S. (2001). Low vitamin $E$ in diet reduces stress resistance of gilthead seabream (Sparus aurata) juveniles. Fish and Shellfish Immunology, 11, 473-490. DOI: $10.1006 /$ fsim.2000.0324 
Montero, D., Robaina, L., Caballero, M.J., Gines, R. \& Izquierdo, M.S. (2005). Growth, feed utilization and flesh quality of European sea bass (Dicentrarchus labrax) fed diets containing vegetable oils: a time course study on the effect of a re-feeding period with $100 \%$ fish oil diet. Aquaculture, 248, 121-134. DOI: 10.1016/j.aquaculture.2005.03.003

Mourente, G. \& Bell, J.G. (2006). Partial replacement of dietary fish oil with blends of vegetable oils (rapeseed, linseed and palm oils) in diets for European seabass (Dicentrarchus labrax L.) over a long term growth study: effects on muscle and liver fatty acid composition and effectiveness of a fish oil finishing diet. Comparative Biochemistry and Physiology Part B, 145, 389-399.

DOI: 10.1016/j.cbpb.2006.08.012

Ng, W-K., Chong, C-Y., Wang, Y. \& Romano, N. (2013). Effects of dietary fish and vegetable oils on the growth, tissue fatty acid composition, oxidative stability and vitamin $\mathrm{E}$ content of red hybrid tilapia and efficacy of using fish oil finishing diets. Aquaculture, 372-375, 97-110. DOI: 10.1016/j.aquaculture.2012.10.030

Ouraji H., Shabanpur B., Abediankenari A., Shabani A., Nezami A., Sudagar M. \& Faghani S. (2009). Total lipid. fatty acid composition and lipid oxidation of Indian White shrimp (Fenneropenaeus indicus) fed diets containing different lipid sources. Journal of the Science of Food and Agriculture, 89(6), 993-997. DOI: 10.1002/jsfa.3545

Regost, C., Arzel, J., Robin, J., Roselund, G. \& Kaushik, S.J. (2003). Total replacement of fish oil by soybean or oil with return to fish oil in turbot (Psetta maxima) 1. Growth performance, flesh fatty acid profile and lipid metabolism. Aquaculture, 217, 465-482. DOI: $10.1016 / S 0044-8486(02) 00259-4$

Sahan, A., Eroldogan, O.T., Kurutas, E.B., Yilmaz, H.A. \& Demirkale, I. (2016). The effects of cottonseed oil as alternative to fish oil on oxidative stress biomarkers and some hematological parameters in European sea bass (Dicentrarchus labrax L.). Fresenius Environmental Bulletin, 25(7), 2639-2645

Senso L., Suarrez M.D., Ruiz-Cara T. \& Garcira-Gallego M. (2007). On the possible effects of harvesting season and chilled storage on the fatty acid profile of the fillet of farmed gilthead sea bream (Sparus aurata) Food Chemistry, 101, 298-307. DOI: 10.1016/j.foodchem.2006.01.036

Sonu, B. (2018). Fish-oil finishing diets: A strategy to recover long-chain polyunsaturated fatty acids in fish previously fed alternative dietary lipids. International Journal of Life Sciences, 6(2), 466-472

Stancheva M., Merdzhanova A., Dobreva D.A. \& Makedonski L. (2014). Common carp (Cyprinus carpio) and European catfish (Silurus glanis) from Danube River as sources of fat soluble vitamins and fatty acids, Czech Journal of Food Sciences, 32, 16-24. DOI: $10.17221 / 31 / 2013-C J F S$

Şahan, A., Yilmaz, H.A. \& Eroldogan, O.T. (2017). Determination of hematological status as health indicator of European sea bass (Dicentrarchus labrax L.) fed different dietary fatty acids. Turkish Journal of Agriculture - Food Science and Technology, 5(4), 445-450.

DOI: 10.24925/turjaf.v5i4.445-1106

Thanuthong, T., Francis, D.S., Senadheera, S.P.S.D., Jones, P.L. \& Turchini, G.M. (2011). Fish oil replacement in rainbow trout diets and total dietary PUFA content: I) effects on feed efficiency, fat deposition and the efficiency of a finishing strategy. Aquaculture, 320(1), 82-90. DOI :10.1016/j.aquaculture.2011.08.007

Torstensen, B.E., Li, Ø. \& Frøyland, L. (2000). Lipid metabolism and tissue composition in Atlantic salmon (Salmo salar L.)-effects of capelin-, palmand oleic acid enriched sunflower oil as dietary lipid sources. Lipids, 35, 653-664. DOI: 10.1007/s11745-000-0570-6

Turan, H., Sönmez, G. \& Kaya, Y. (2007). Fatty acid profile and proximate composition of the thornback ray (Raja clavata, L. 1758) from the Sinop coast in the Black Sea. Journal of Fisheries Sciences, 1(2), 97-103. DOI: 10.3153/jscom.2007012

Turchini, G.M., Francis, D.S. \& De Silva, S.S. (2006). Modification of tissue fatty acid composition in Murray cod (Maccullochella peelii peelii, Mitchell) resulting froma shift from vegetable oil diets to a fish oil diet. Aquaculture Research, 37, 570-585. DOI: $10.1111 / j .1365-2109.2006 .01465 . x$

Ulbricht, T. \& Southgate, D. (1991). Coronary heart disease: seven dietary factors, The Lancet, 338, 985-992. DOI: 10.1016/0140-6736(91)91846-M

URL2, (2016). HealthyLiving, HealthyEating, Healthy Diet Goals, Fish and Omega-3 Fatty Acids. Retrivied from

http://www.heart.org/HEARTORG/HealthyLiving/HealthyEating/ HealthyDietGoals/Fish-and-Omega-3-

FattyAcids_UCM_303248_Article.jsp\#. jtw499I-M8, In: Dagtekin, B.B.G. Misir, G.B., Kutlu, S. \& Basturk, O., (2017). Mediterranean Fisheries and Aquaculture Research, 1(1), 2-14.

Yildiz, M., Köse, I., Issa, G. \& Kahraman, T. (2015). Effect of different plant oils on growth performance, fatty acid composition and flesh quality of rainbow trout (Oncorhynchus mykiss). Aquaculture Research, 46, 28852896. DOI: 10.1111/are.12441

Yıldız, M., Eroldoğan, T.O., Ofori-Mensah, S., Engin, K. \& Baltacı, M.A. (2018). The effects of fish oil replacement by vegetable oils on growth performance and fatty acid profile of rainbow trout: Re-feeding with fish oil finishing diet improved the fatty acid composition. Aquaculture, 488, 123-133. DOI: 10.1016/j. aquaculture.2017.12.030

Yu, J., Chang, J., Niu, H., Hu, Z. \& Han, Y. (2018). Effect of variation in the dietary ratio of linseed oil to fish oil on growth, body composition, tissues fatty acid composition, flesh nutritional value and immune indices in Manchurian trout, Brachymystax lenok. Aquaculture Nutrition, 1-11. DOI: $10.1111 /$ anu.12863 